\title{
Cryptococcosis: Its Prevalence and Clinical Presentation among Hiv Positive and Negative Patients in Rims, Manipur
}

\author{
Phangreichon Lungran ${ }^{1}$, Aribam Vijaya Devi ${ }^{2}$, Waikhom Shashi Singh ${ }^{3}$, \\ S Damroulien ${ }^{4}$, Henkhoneng Mate ${ }^{5}$, Achuina Golmei ${ }^{6}$ \\ ${ }_{1,2,3,4,5,6}$ Department of Microbiology, RIMS, Manipur, India
}

\begin{abstract}
Cryptococcus neoformans, the causative fungal agent of cryptococcosis remain a common cause of infectious morbidity and mortality, especially among HIV-positive patients living in Sub-Saharan Africa and South-East Asia. This study was undertaken to evaluate the prevalence and clinical presentation of Cryptococcus infections among HIV positive and negative patients in RIMS, Manipur. Specimens like CSF, sputum, urine, blood, tissue biopsy or aspirates from clinically suspected cryptococcosis cases from RIMS hospital, were subjected to mycological examination. Out of the 48 patients enrolled for the study, Cryptococcus spp were isolated from 16 (33.33\%) patients. Among these 16 cryptococcosis patients, majority of them presented with cryptococcal meningitis 13 (81.25\%), while 1 (6.25\%) patient each presented with cryptococcal lymphadenitis, disseminated cutaneous cryptococcosis and osseous cryptococcosis respectively. Also, of these 16 cryptococcosis patients, 14 (87.5\%) were HIV positive. Among these HIV positive, sexual transmission with $64.29 \%$ forms the commonest route of transmission, followed by intravenous drug injection with 35.71\%. Out of these $14 \mathrm{HIV}$ positive patients, 11 (78.57\%) were on ART. This study shows high prevalence of cryptococcosis in our region with the most common presentation being cryptococcal meningitis. Also, there was high incidence of cryptococcosis among HIV-infected patients.
\end{abstract}

Key words: Cryptococcosis, Cryptococcus neoformans, HIV

\section{Introduction}

Cryptococcus neoformans is an encapsulated yeast, ubiquitously found worldwide in soil contaminated with bird droppings mainly of pigeons, roosting sites and decaying woods and vegetables. The infectious propagules of this fungus from these environments may be the source of human infections [1,2]. Cryptococcus neoformans, the causative agent of cryptococcosis, can cause life threatening infections with predilection for brain and meninges, occasionally lungs and skin. Although very rare, it may involve in other organs such as the heart, liver, spleen, kidneys, thyroid, lymphnodes, adrenal glands, eyes, genitourinary tract, gastrointestinal tract and abdominal, bones and joints [3]. The emerging menace of cryptococcosis has assumed global proportion over the years due to climate changes and the worldwide spread of HIV infection as well as the increasing number of patients with impaired immunity as a result of cancer chemotherapy, diabetes mellitus, sarcoidosis, steroids, renal transplant or inherited immune defects. It also causes infection less commonly in immunocompetent hosts [4,5]. Although effective treatment for HIV disease has led to significant decrease in the incidence of cryptococcal meningitis (CM) in the western countries, it continues to be the common cause of infectious morbidity and mortality especially among the HIV-positive patients living in Sub-Saharan Africa and South-East Asia [6,7,8,9].

Due to the high prevalence of HIV infection in our state Manipur [10], a large percentage of the population continues to be at risk for infection with indigenous Cryptococcus strains. This study was undertaken to evaluate the prevalence and clinical presentation of Cryptococcus infection among both the HIV positive and negative patients in Regional Institute of Medical Sciences (RIMS) hospital, Manipur.

\section{Materials and methods}

The study was conducted in the Microbiology Department, RIMS, Imphal Manipur, with a study period of one and a half years starting from November 2011 to October 2013. Patients enrolled in the study were taken informed consent and in the case of minor, informed consent were taken from parents. This was a Cross sectional study carried out under the approval of the institutional ethical committee, RIMS.

The study consist of 67 samples, collected from 48 clinically suspected cryptococcosis cases in RIMS hospital and were subjected to mycological examination. The specimens included CSF, sputum, urine, blood, tissue biopsy or aspirates depending upon the site of involvement i.e. from superficial lesions to deep seated infections. Multiple samples were taken from a single patient. The specimens were subjected to examination by India ink preparation, Meyer's mucicarmine stains, Giemsa stains (Fig.1) and antigen detection (CALAS, Meridian Diagnostics, Cincinnati, Ohio). The specimens were further confirmed by fungal culture on Sabouraud 
dextrose agar (Emmon's modification with antibiotics chloremphenicol) and laboratory-prepared Bird seed agar plates (BSA) which showed mucoid colonies (Fig.2). Isolates were identified as C. neoformans by growth at $37^{\circ} \mathrm{C}$, urease test, inositol assimilation, subculture on L-canavanine glycine bromothymol blue (CGB) and creatinine dextrose bromothymol blue thymine (CDBT) medium detected by color reaction. The identity of the isolates were also confirmed by automated Vitek 2 yeast identification system [bioMerieux Inc., (Durham, NC) USA]. The datas so collected were processed using SPSS version 16.0.

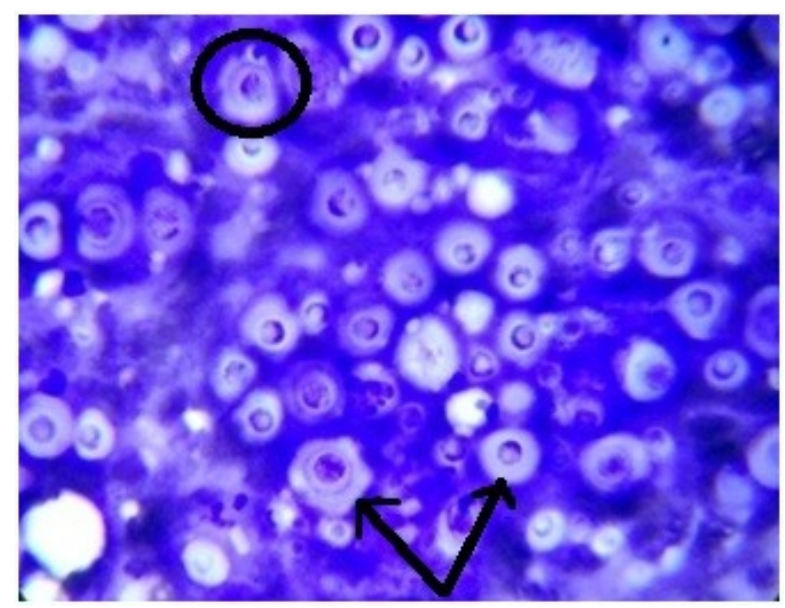

Figure 1: Encapsulated Cryptococcus in skin aspirate's Giemsa stain(x1000)

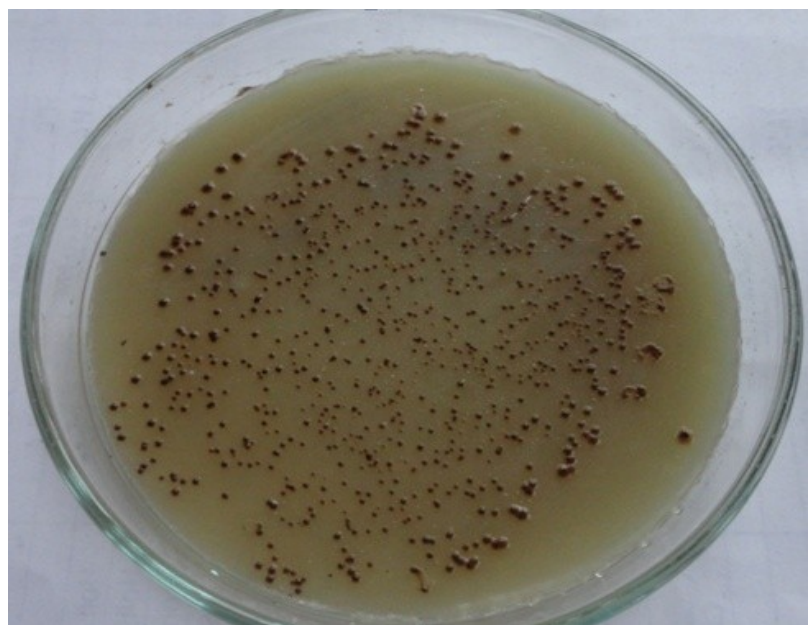

Figure 2: BSA mucoid brown colonies of Cryptococcus spp

\section{Results}

Out of the total 67 clinical samples taken, $16(23.88 \%)$ were CSF, 15 (22.39\%) were blood, 17 (25.37\%) urine, 16 (23.88\%) sputum and 3 (4.48\%) others (i.e. aspirate of skin, lymphnode \& scalp). Out of 48 patients enrolled in the study, Cryptococcus spp were isolated from 16(33.33\%). Among these 16 cryptococcosis patients, majority of them presented with cryptococcal meningitis $13(81.25 \%)$, while 1 (6.25\%) patient each were presented with cryptococcal lymphadenitis, disseminated cutaneous cryptococcosis and osseous cryptococcosis respectively. The ratio of male to female cryptococcosis patients was 4.3:1 (TABLE $1)$. 


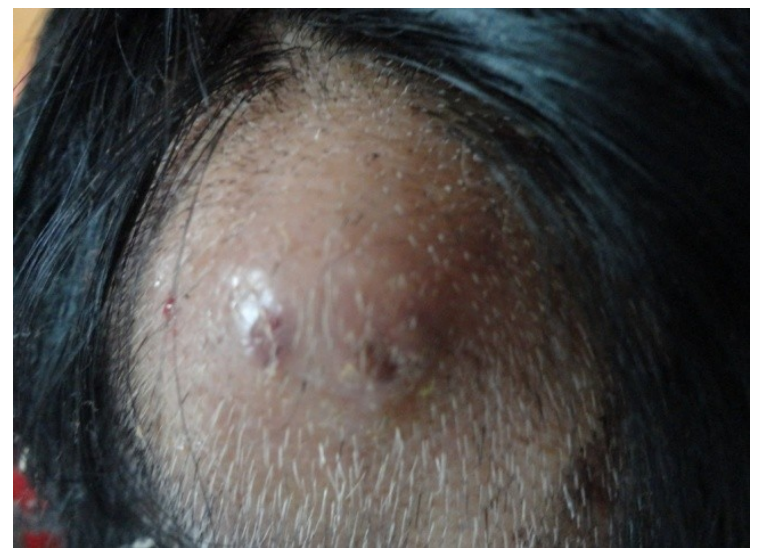

Figure 3: Swelling over the right side of scalp in osseous cryptococcosis patient.

Table 1: Distribution of cryptococcosis among culture positive patients

\begin{tabular}{|l|c|c|c|l|}
\hline Cryptococcosis & Male & Female & Total & Percentage \\
\hline Cryptococcal Meningitis & 11 & 2 & 13 & $81.25 \%$ \\
\hline Cryptococcal Lymphadenitis & 1 & 0 & 1 & $6.25 \%$ \\
\hline Disseminated cutaneous Cryptococcosis & 0 & 1 & 1 & $6.25 \%$ \\
\hline Osseous cryptococcosis & 1 & 0 & 1 & $6.25 \%$ \\
\hline Total & $13(71.43 \%)$ & $3(28.57 \%)$ & 16 & $100 \%$ \\
\hline
\end{tabular}

Among 16 cryptococcosis patients, $14(87.5 \%)$ were HIV positive. And among these HIV positive patients, route of transmission through sexual was $9(64.29 \%)$ and $5(35.71 \%)$ was through intravenous drug injection. Out of 14 HIV positive patients, 11 (78.57\%) were on ART (Table 2)

Table 2: HIV prevalence, route of infection and ART status among the culture positive patients in relation to age

\begin{tabular}{|c|c|c|c|c|c|}
\hline \multirow{2}{*}{ Age group } & \multirow{2}{*}{ No. of patients } & \multirow{2}{*}{ HIV positive } & \multicolumn{2}{|c|}{ Route of infection } & \multirow{2}{*}{ On ART } \\
\cline { 3 - 5 } & & & STI & IVDU & \\
\hline $1-20$ & 1 & 0 & 6 & 0 & 0 \\
\hline $21-40$ & 11 & 11 & 1 & 5 & 9 \\
\hline $41-60$ & 1 & 1 & 2 & 0 & 0 \\
\hline $61-80$ & 3 & 2 & $9(64.29 \%)$ & $5(35.71 \%)$ & $11(78.57 \%)$ \\
\hline Total & 16 & $14(87.5 \%)$ &
\end{tabular}

*STI-Sexually Transmitted Infection; IVDU-Intravenous Drug Use.

\section{Discussion}

Cryptococcus neoformans has become one of the most prevalent causes of fungal disease affecting more than one million people and causing at least 500,000 deaths annually worldwide due to HIV-associated cryptococcosis [11]. It is suggested that cryptococcosis one of the AIDS defining infections considered as "sleeping disease" became an "awakening giant" within a couple of years [12]. Manipur, located in the north eastern corner of India is one of the high prevalent states of HIV infection [10].

In this present study, the prevalence of crytococcosis was observed to be high (TABLE 1). This finding is related to the study taken by Uma B [13] which reported increasing trend of crytococcosis with the increase in the prevalence of HIV/AIDS over the years from $20 \%$ to $49 \%$. This high prevalence of crytococcosis may reflect an enhanced clinical awareness, improved diagnostic capability and also increasing prevalence of HIV/AIDS. Cryptococcal meningitis was the most common presentation $(81.25 \%)$ followed by cryptococcal lymphadenitis, disseminated cutaneous cryptococcosis and osseous cryptococcosis with $6.25 \%$ each respetively. The high prevalence of cryptococcal meningitis patients in this study concurs with the findings reported in some studies in the HIV positive patients $[14,15]$. And also the prevalence of male higher than the female in this present study was comparable to the reports in other studies [16,17,18]. There is hypothesis that differential interaction between Cryptococcus and macrophages within different gender environments and hormone contribute to the increased prevalence of cryptococcosis in males [19].

The percentage of age distribution among cryptococcosis patients was maximum in the age group of $21-40$ yrs $(68.75 \%)$ which is statistically significant $(\mathrm{p}=0.02)$ in this study. The maximum distribution of the patients in age group 20-40 was also observed by Lakshmi V et al. [17] with a mean of 31 years. The youngest patient among the study population was a 6 years old boy who presented with cryptococcal lymphadenitis and was HIV negative. A case of disseminated cutaneous cryptococcosis with pulmonary tuberculosis was found in 
a HIV positive ART naïve female patient where Cryptococcus was isolated from skin aspirates, blood, bone marrow and urine. In this current study, an immunocompetent male patient presented with osseous cryptococcosis on the right scalp. All of them responded well to antifungal therapy. This present study showed that the prevalence of cryptococcosis in HIV positive patients were high (87.5\%). The high prevalence of HIV positive in relation to cryptococcosis patients was comparable with the finding of Babbyngttonn KDS et al. [16] and the predominance of cryptococcosis in the third and fourth decades of life coincides with the age range where HIV is most prevalent. Among these HIV positive patients, transmission through sexual contact was higher than through intravenous drug injection. Linda M [20] suggested that sexual transmission of HIV is by far the most common mode of transmission globally which is similar with the finding of our study. Out of these 14 HIV positive patients, 11 (78.57\%) were on ART (Table 2). The reason for high prevalence of HIV positive cryptococcosis on ART have not been studied here but maybe due to non compliance of the patients or non availability of ART. Manipur presently reporting as high prevalence HIV positive state in India, coupled with its subtropical climate with rich flora and fauna may also be the reason for high prevalence of cryptococcosis. Thus, further study on the impact of ART and exploration of the effect of location and environment on the risk of cryptococcal infection may bring more insight to the current problem.

\section{Conclusion}

This study shows that cryptococcosis is highly prevalent especially among HIV positive patients and common presentation being cryptococcal meningitis. In general, people should be made aware of the disease and physicians should have high clinical suspicion especially to HIV positive patients to initiate prompt investigation and treatment for better outcome.

\section{References}

[1]. KV Ramana, MV Kumar, SD Rao, R Akhila, Sandhya, et al. Pulmonary Cryptococcosis secondary to Bronchial Asthma Presenting as Type I Respiratory Failure- A Case Report with Review of Literature. Virol Mycol. 1(107), 2012, doi:10.4172/21610517.1000107

[2]. CS Tania, HE David. Ecology of Cryptococcus neoformans.Rev Iberoam Micol, 14, 1997, 42-43.

[3]. C Yat, M Sunny, J B Paxton, C Huiming, O A Adrian, W K James at el. Characterization of Environmental Sources of the Human and Animal Pathogen Cryptococcus gattii in British Columbia, Canada, and the Pacific Northwest of the United States. Appl. Environ.Microbiol ,73 (5), 2007, 1433-1443.

[4]. K Lemmer, D Naumann, B Raddatz, K Tintelnot. Molecular typing of Cryptococcus neoformans by PCR fingerprinting, in comparison with serotyping and Fourier transform infrared-spectroscopy-based phenotyping. Med Mycol. 42 (2):2004; $135-147$.

[5]. M Cogliati. Global Molecular Epidemiology of Cryptococcus neoformans and Cryptococcus gattii: An Atlas of the Molecular Types. Hindawi Publishing Corporation. 2013, Article ID 675213, 23 pages http://dx.doi.org/10.1155/2013/675213.

[6]. SA Mirza, M Phelan, D Rimland. The changing epidemiology of cryptococcosis: an update from population-based active surveillance in 2 large metropolitan areas, 1992-2000. Clinical Infectious Diseases. 36(6), 2003, 789-794.

[7]. ELJR Van, AME Walenkamp, MM Lipovsky. Declining number of patients with cryptococcosis in the Netherlands in the era of highly active antiretroviral therapy. AIDS. 14(17), 2000, 2787-2788.

[8]. JN Jarvis, A Boulle, A Loyse. High ongoing burden of cryptococcal disease in Africa despite antiretroviral roll out. AIDS.23(9), 2009, 1182-1183.

[9]. JR Harris, MD Lindsley, S Henchaichon. High prevalence of cryptococcal infection among HIV-infected patients hospitalized with pneumonia in Thailand. Clinical Infectious Diseases. 54(5), 2012, 43-50.

[10]. National AIDS Control Organisation (NACO) HIV sentinels surveillance 2010-2011 in India.

[11]. C Massimo. Global Molecular Epidemiology of Cryptococcus neoformans and Cryptococcus gattii. An Atlas of the Molecular Types Scientifica 2013; Article ID 675213,23pages http://dx.doi.org/10.1155/2013/675213

[12]. V Baradkar, M Mathur, S Kumar, M Rathi. Prevalence and clinical presentation of Cryptococcal meningitis among HIV seropositive patients. Indian J Sex Transm Dis. 30, 2009, 19-22.

[13]. B Uma. Progress in diagnosis of opportunistic infections in HIV/AIDS. Indian J Med Res 2005; 395-406

[14]. R Thakur, S Sarma, S Kushwaha. Prevalence of HIV-associated cryptococcal meningitis and utiity of microbiological determinants for its diagnosis in a tertiary care center. Indian J Pathol Microbiol. 51, 2008, 212-4.

[15]. P Mwaba, J Mwansa, C Chintu, J Pobee, M Scarborough, S Portsmouth at el. Clinical presentation, natural history, and cumulative death rates of 230 adults with primary cryptococcal meningitis in Zambian AIDS patients treated under local conditions. Postgrad Med J. 77, 2001,769-773.

[16]. KDS Babbyngttonn, KF Ana, DSB Amaury, DLS Ivanete, OS Lucilaide, SDS Mirlane at el. Characterization of clinical isolates of the Cryptococcus neoformans-Cryptococcus gattii species complex from the Amazonas State in Brazil. Rev Iberoam Micol. 29(1), 2012, 40-43.

[17]. V Lakshmi, T,Sudha, VD Teja, P Umabala. Prevalence of central nervous system cryptococcosis in human immunodeficiency virus reactive hospitalized patients. Indian J Med Microbiol. 25(2), 2007, 146-9.

[18]. Capoor MR, Nair D, Deb M, Gupta B, Aggarwal P. Clinical and mycological profile of cryptococcosis in a tertiary care hospital. Indian J Med Microbiol. 25(4), 2007, 401-4.

[19]. EE McClelland, LM Hobbs, J Rivera, A Casadevall, WK Potts et al. The Role of Host Gender in the Pathogenesis of Cryptococcus neoformans Infections. PLoS ONE. 8(5), 2013, e63632. Doi: 10.1371/ journal.pone.0063632.

[20]. M Linda. The global epidemiology of HIV/AIDS. British Medical Bulletin. 58, 2001, 7-18. 